\title{
Traffic Performance of Crawler from Viewpoint of Energy Analysis
}

\author{
K. Kogure, N.Moriyama and T.Shogaki \\ Department of Civil Engineering \\ National Defense Academy \\ 1-10-20 Hashirimizu \\ Yokosuka, Kanagawa, Japan 239
}

\begin{abstract}
In studies concerned with soil crawler interaction, it is known that there exist interdependent relationship between soil and crawler. This paper deals with a method of basic energy analysis for crawler motion on the ground. Six types of motion resistances acting on the running gear of a crawler are considered and the relationship between the driving force, drawbar pull and motion resistances are investigated. The conservation of energies between the input energy, drawbar pul1 energy, compaction resistance energy, bulldozing resistance energy, two slip energies and track rotation resistance energy are analysed for unit travelling and unit time.
\end{abstract}

\section{INTRODUCTION}

The principle of energy conservation is one of the basic laws of physics. Based on the principle, some investigations on the analysis between the running gear of vehicle and soil were reported[1,2,3]. Authors considered on the soil thrust and motion resistance exerted by tracked vehicle $[4,5]$. The paper is practically considered the basic ideas of the energy conservation between a crawler type tractor with rigid track and soil. Six types of motion resistance acting to running gear of a crawier tractor motion on soil are introduced into the analysis and the energy equilibrium equation between the input, output and motion resistance energies for the motion of running gear is considered.

The performance of running gear with rigid track is evaluated in terms of the output energy that can be developed in view of the track contact surface characteristics and soil properties. The amount of input energy that can be developed for production of drawbar pull is intimately tied into the properties of soil and slippage of track. The input energy per unit travelling distance of crawler tractor increases with the increasing slippage. The output energy per unit time of crawler tractor decreases with slippage and the maximum output energy occures at no slippage.

\section{SLIPPAGE AND FORCES ACTING RUNNING GEAR}

The slippage occures between the running gear and soil when the crawler is travel1ing under pulling load. The slip ratio $i$ at pulling state is given as

$$
i=\frac{v_{t}-v}{v_{t}}=1-\frac{v}{v_{t}}
$$

where, $i: s l i p$ ratio, $v_{t}$ :rotation speed of track belt, $V:$ horizontal vehicle travelling speed. The slip ratio can be considered as ratio of horizontal deformation $j$ to the contact distance $x$. Therefore, the soil deformation $j$ under slipping track can be 
represented by $j=i x$, where, $j:$ so $i 1$ deformation, $x$ :contact distance from the front of track. The total slippage of the running gear is $i L$ when the crawler is travelling with slip ratio $i$, where, $L$ is the contact length.

Fig. 1 shows the forces acting on the running gear. In Fig. 1, $F_{i}$ :driving force, $F_{0}$ :drawbar pul1, $F_{b s}:$ soil shear force under one track belt, $F_{S S}$ : soil shear force on one side of track belt, $F_{c}$ : compaction resistance for one track belt, $F_{b}:$ bulldozing resistance for one track belt, $F_{a}$ :adhesion resistance on one side of running gear, $F_{r}$ :rotation resistance of one track belt.

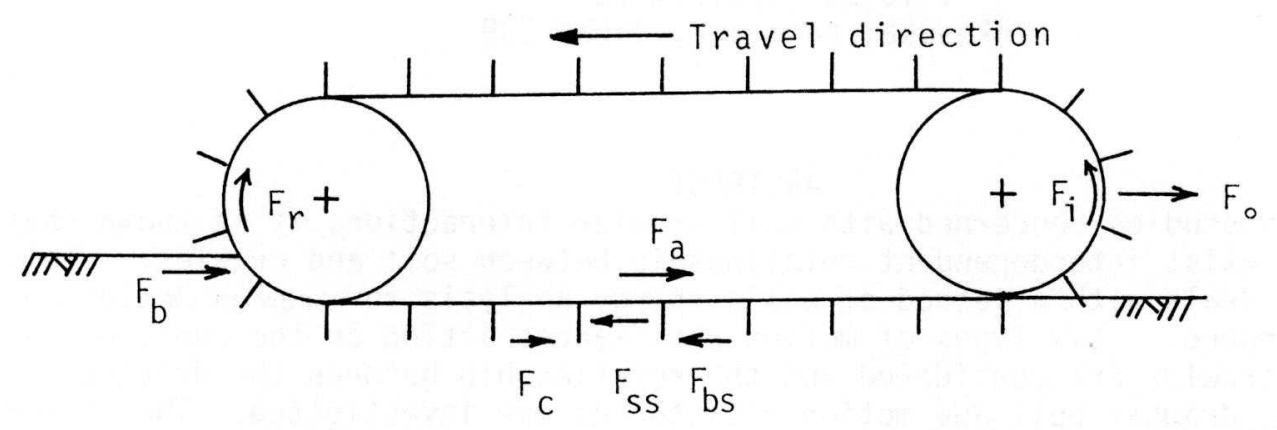

Fig. 1 Forces acting on running gear

The total driving force of crawler tractor is the sum of $2 F_{r}, 2 F_{b s}$ and $4 F_{S S}$. The balance of the foeces is given as follows

$$
2 F_{i}=2 F_{b s}+4 F_{S S}+2 F_{r}
$$

The soil thrust exerted by the tractor is given as ( $2 \mathrm{Fbs}+4 \mathrm{Fss}$ ) and the motion resistance is represented by $\left(2 F_{c}+2 F_{b}+4 F_{a}\right)$. The relationship between the forces acting on travelling tractor on horizontal plane is given as

$$
2 F_{b s}+4 F_{s s}=F_{o}+2 F_{c}+2 F_{b}+4 F_{a}
$$

Equation (3) shows that the drawbar pull is defined by subtracting the motion resistance from the soil thrust.

\section{ENERGY CONSERVATION FOR RUNNING GEAR MOTION}

The energy equilibrium for running gear motion can be represented based on the acted forces as follows

$$
E_{i}=E_{0}+E_{c}+E_{b}+E_{b s}+E_{S S}+E_{a}+E_{r}
$$

where, $E_{j}$ :input energy, $E_{0}$ :drawbar pull energy, $E_{C}$ :compaction energy, $E_{b}$ :bulldozing energy, $E_{b s}$ :bottom slip energy, $E_{S s}$ : side slip energy, $E_{a}$ :adhesion resistance energy, $E_{r}$ :rotation resistance energy.

\subsection{Input Energy $E_{i}$}

The input energy $E_{j}$ is defined as the effective input energy and is inputted to the sprocket by the driving force $2 F_{i}$. The input energy for track motion of tractor is represented as follows

$$
E_{i}^{\prime}=2 F_{i} L \quad(\mathrm{kgf} \cdot \mathrm{cm})
$$


where, $E_{i}^{\prime}:$ input energy when the track belt rotates the distance $L, L:$ contact length. The tractor travelling distance $L^{\prime}$ when the track belt rotates $L$ is $L^{\prime}=(1-i) L$.

Therefore,

$$
\bar{E}_{i}=\frac{E_{i}^{\prime}}{L^{\prime}}=\frac{2 F_{i} L}{(1-i) L}\left(=\frac{2 F_{i}}{1-i}\right) \quad(\mathrm{kgf} \cdot \mathrm{cm} / \mathrm{cm})
$$

where, $\bar{E}_{j}$ : input energy when the tractor is advanced unit distance(input energy per unit traveliling distance). The input energy can be expressed as follows

$$
\dot{E}=2 F_{i} V_{t} \quad(\mathrm{kgf} \cdot \mathrm{cm} / \mathrm{sec})
$$

where, $\dot{E}_{j}$ : input energy when the tractor travels per unit time(input energy per unit time).

\subsection{Drawbar Pul1 Energy E。}

For the tractor motion over the distance $L^{\prime}$ with the slip ratio $i$, the drawbar pull energy $E_{0}$ will be defined as

$$
E_{0}^{\prime}=F_{0} L^{\prime}=F_{0}(1-i) L \quad(\mathrm{kgf} \cdot \mathrm{cm})
$$

Therefore,

$$
\begin{array}{lll}
\bar{E}_{0}=\frac{E_{0}^{\prime}}{L^{\prime}}=\frac{F_{0}(1-i) L}{(1-i) L}\left(=F_{0}\right) & (\mathrm{kgf} \cdot \mathrm{cm} / \mathrm{cm}) \\
\dot{E}_{0}=\frac{E_{0}^{\prime}}{\frac{L}{V_{t}}}=F_{0}(1-i) V_{t} & (\mathrm{kgf} \cdot \mathrm{cm} / \mathrm{sec})
\end{array}
$$

where, $\bar{E}_{\circ}$ :drawbar pull energy per unit travelling distance, $E_{\circ}^{\prime}$ :drawbar pull energy per unit time.

\subsection{Compaction Energy $E_{C}$}

The compaction energy $E_{C}$ identifies the energy reqired to compact the soil as a result of track motion. Here, it is assumed that the relation between the contact pressure $p$ and sinkage $z$ of the track plate can be represented as follows[6]

$$
p=k z^{n}
$$

where, $k$ and $n$ are experimental constants. The mean contact pressure between track and soil is written as

$$
p=\frac{W}{2 B L}
$$

where, w:tractor weight, B:contact width. The compaction energy of soil per track contact area (2BL) CE may be expressed as 


$$
C E=2 B L \int_{0}^{z_{0}} p d z=\frac{W}{n+1}\left(\frac{W}{2 B L k}\right)^{1 / n} \quad(\mathrm{kgf} \cdot \mathrm{cm})
$$

where, $z_{0}$ :sinkage of track which is obtained from eq.(11) and (12). The compaction energy $C E$ can be equated to the work done in overcoming compaction resistance $F_{C}$ and from $C E=2 F_{C} L$,

$$
F_{C}=\frac{W}{2 L(n+1)}\left(\frac{W}{2 B L k}\right)^{1 / n}
$$

When the compaction resistance force $F_{C}$ is obtained from eq. (14), the compaction energy is given as

$$
E_{C}^{\prime}=2 F_{C} L^{\prime}=2 F_{C}(1-i) L \quad(\mathrm{kgf} \cdot \mathrm{cm})
$$

and

$$
\bar{E}_{C}=\frac{E_{C}^{\prime}}{L^{\prime}}=\frac{2 F_{C}(1-i) L}{(1-i) L}\left(=2 F_{C}\right) \quad(\mathrm{kgf} \cdot \mathrm{cm} / \mathrm{cm})
$$

or

$$
\dot{E}_{C}=\frac{E_{C}^{\prime}}{L / V_{t}}=2 F_{C}(1-i) V_{t} \quad(\mathrm{kgf} \cdot \mathrm{cm} / \mathrm{sec})
$$

where, $E_{C}^{\prime}$ : compaction energy when the track belt rotates the distance $L, \bar{E}_{C}$ :compaction energy per unit travel1ing distance, $\dot{E}_{C}$ :compaction energy per unit time.

\subsection{Bulldozing Energy $E_{b}$}

In predicting the bulldozing resistance, it is assumed that it is equivalent to the horizontal force of the passive earth pressure acting on a vehicle front. Here, the bulldozing resistance $\mathrm{F}_{\mathrm{b}}$ was estimated using the passive earth pressure theory. The bulldozing energy can be estimated when the force $\mathrm{Fb}$ is obtained as follows

$$
E_{b}^{\prime}=2 F_{b} L^{\prime}=2 F_{b}(1-i) L \quad(\mathrm{kgf} \cdot \mathrm{cm})
$$

and

$$
\bar{E}_{b}=\frac{E_{b}^{\prime}}{L^{\prime}}=\frac{2 F_{b}(1-i) L}{(1-i) L}\left(=2 F_{b}\right) \quad(\mathrm{kgf} \cdot \mathrm{cm} / \mathrm{cm})
$$

or

$$
\dot{E}_{b}=\frac{E_{b}^{\prime}}{L / V_{t}}=2 F_{b}(1-i) V_{t} \quad(\mathrm{kgf} \cdot \mathrm{cm} / \mathrm{sec})
$$

where, $E_{b}^{\prime}$ :bulldozing energy when track belt rotates the distance $L, \bar{E}_{b}$ and $E_{b}^{\prime}$ : bulldozing energies per unit travelling distance and per unit time respectively.

\subsection{Bottom S1ip Energy $E_{b s}$}

The bottom slip energy $E_{\text {hs }}$ refers to the energy loss due to slippage between the bottom of track and soil. The force by bottom slippage Fbs may be estimated as 
follows

$$
F_{b s}=B L(c+p \tan \varnothing)
$$

where, c:cohesion of soil, $\phi$ :internal friction angle of soil. Therefore, the bottom slip energy Ebs is obtained as

$$
E_{b s}^{\prime}=2 F_{b s} i 1 \quad(\mathrm{kgf} \cdot \mathrm{cm})
$$

and

$$
\bar{E}_{b s}=\frac{E_{b s}^{\prime}}{L^{\prime}}=\frac{2 F_{b s} i L}{(1-i) L}\left(=2 F_{b s} \frac{i}{1-i}\right) \quad(\mathrm{kgf} \cdot \mathrm{cm} / \mathrm{cm})
$$

or

$$
\dot{E}_{b s}=\frac{E_{b s}^{\prime}}{L / V_{t}}=2 F_{b s} i V_{t} \quad(\mathrm{kgf} \cdot \mathrm{cm} / \mathrm{sec})
$$

where, Ébs:bottom slip energy when track belt rotates the distance $L, \bar{E}_{b s}$ and $\dot{E}_{b s}$ : bottom slip energies per unit travelling distance and per unit time respectively.

\subsection{Side STip Energy $\mathrm{E}_{\mathrm{SS}}$}

The side slip energy $E_{S S}$ refers to the energy loss due to slip between the side of track and soil. The side slip force Fss may be estimated as follows

$$
F_{S S}=H L(c+\sigma \tan \phi)
$$

where, $H$ :grouser high, $\sigma:$ normal stress. The side slip energy is given as

$$
E_{S S}^{\prime}=4 F_{S S} i L \quad \text { (kgf.cm) }
$$

and

$$
\bar{E}_{S S}=\frac{E_{S S}^{\prime}}{L^{\prime}}=\frac{4 F_{S S} i L}{(1-i) L} \quad\left(=4 F_{S S} \frac{i}{1-i}\right) \quad(\mathrm{kgf} \cdot \mathrm{cm} / \mathrm{cm})
$$

or

$$
\dot{E}_{S S}=\frac{E_{S S}^{\prime}}{L / V_{t}}=4 F_{S S} i V_{t} \quad(\mathrm{kgf} \cdot \mathrm{cm} / \mathrm{sec})
$$

where, $E_{S S}^{\prime}$ :side slip energy when track belt rotates the distance $L, \bar{E}_{S S}$ and $\dot{E}_{S S}$ : side slip energies per unit travelling distance and per unit time respectively.

\subsection{Adhesion Energy $\mathrm{E}_{\mathrm{a}}$}

When the sinkage of tractor is considerably large, the adhesion resistance force $\mathrm{Fa}$ may be acted between the tractor body and soi7. If the adhesion ca between the tractor body and soil is known, the adhesion resistance force $\mathrm{Fa}_{\mathrm{a}}$ may be expressed as

$$
F_{a}=Z_{0} L \tau_{a}
$$

and the adhesion energy is given as follows

$$
E_{a}^{\prime}=4 F_{a} L^{\prime}=4 F_{a}(1-i) L \quad(\mathrm{kgf} \cdot \mathrm{cm})
$$

and 


$$
\bar{E}_{a}=\frac{E_{a}^{\prime}}{L^{\prime}}=\frac{4 F_{a}(1-i) L}{(1-i) 1} \quad\left(=4 F_{a}\right) \quad(\mathrm{kgf} \cdot \mathrm{cm} / \mathrm{cm})
$$

or

$$
\dot{E}_{a}=\frac{E_{a}^{\prime}}{L / V_{t}}=4 F_{a}(1-i) V_{t} \quad(\mathrm{kgf} \cdot \mathrm{cm} / \mathrm{sec})
$$

where, $E_{a}^{\prime}$ :adhesion energy when track belt rotates the distance $L, \bar{E}_{a}$ and $\dot{E}_{a}$ :adhesion energy per unit travelling distance and per unit time respectively.

\subsection{Rotation Energy $E_{r}$}

The force due to rotation of track belt is the rotation resistance force $F_{r}$. The force is measured as a part of the driving force of sprocket, but it is does not distribute to the thrust of tractor. The force is one of the resistance force.

The rotation energy is given as follows

$$
E_{r}^{\prime}=2 F_{r} L \quad \text { (kgf.cm) }
$$

and

or

$$
\bar{E}_{r}=\frac{E_{r}^{\prime}}{L^{\prime}}=\frac{2 F_{r} L}{(1-i) L}\left(=2 F_{r} \frac{i}{1-i}\right) \quad(\mathrm{kgf} . \mathrm{cm} / \mathrm{cm})
$$

$$
\dot{E}_{r}=\frac{E_{r}^{\prime}}{L / V_{t}}=2 F_{r} V_{t} \quad(\mathrm{kgf} \cdot \mathrm{cm} / \mathrm{sec})
$$

where, $E_{r}^{\prime}$ :rotation energy when track belt rotates the distance $L, \bar{E}_{r}$ and $\dot{E}_{r}$ :rotation energy per unit travelling distance and per unit time respectively.

\subsection{Energy Conservation for Running Gear}

Based on the definitions of the energies mentioned above, the energy equilibrium of the running gear of crawler tractor motion can be written from eq. (4) as follows,

$$
E_{i}^{\prime}=E_{0}^{\prime}+E_{c}^{\prime}+E_{b}^{\prime}+E_{b s}^{\prime}+E_{s S}^{\prime}+E_{a}^{\prime}+E_{r}^{\prime} \quad(k g f \cdot c m)
$$

or

$$
\bar{E}_{i}=\bar{E}_{0}+\bar{E}_{c}+\bar{E}_{b}+\bar{E}_{b s}+\bar{E}_{s S}+\bar{E}_{a}+\bar{E}_{r} \quad(\mathrm{kgf} \cdot \mathrm{cm} / \mathrm{cm})
$$

or

$$
\dot{\mathrm{E}}_{i}=\dot{\mathrm{E}}_{o}+\dot{\mathrm{E}}_{c}+\dot{\mathrm{E}}_{\mathrm{b}}+\dot{\mathrm{E}}_{\mathrm{bs}}+\dot{\mathrm{E}}_{s s}+\dot{\mathrm{E}}_{\mathrm{a}}+\dot{\mathrm{E}}_{r}
$$

4. DISCUSSIONS

Some discussions will be made the proposed energy conservation based on the calculated results for a tractor and soil conditions. The characteristics of crawler tractor used in the calculation of the energy conservation are shown in Table 1 and the properties of soil for calculation are shown in Table 2.

It is assumed that the tractor speed $V_{t}$ is $100 \mathrm{~cm} / \mathrm{sec}$. The horizontal force of the passive earth pressure is used as the bulldozing resistance. 
Table 1 Characteristics of crawler

\begin{tabular}{l|r}
\hline Total weight W & $16000 \mathrm{kgf}$ \\
Contact length L & $280 \mathrm{~cm}$ \\
Track width B & $50 \mathrm{~cm}$ \\
Grouser hight H & $5 \mathrm{~cm}$ \\
Contact pressure p & $0.571 \mathrm{kgf} / \mathrm{cm}^{2}$ \\
\hline
\end{tabular}

Table 2 Soil properties

\begin{tabular}{l|c|c}
\hline & Cohesive soil & Frictional soit \\
\hline Cohesion c (kgf/cm²) & 0.4 & 0 \\
Internal friction angle $\phi($ deg.) & 0 & 33 \\
Adhesion $\tau_{a}\left(\mathrm{kgf} / \mathrm{cm}^{2}\right)$ & 0.2 & 0.1 \\
Constant $k$ & 0.06 & 0.6 \\
Constant $n$ & 0.9 & 1.2 \\
\hline
\end{tabular}

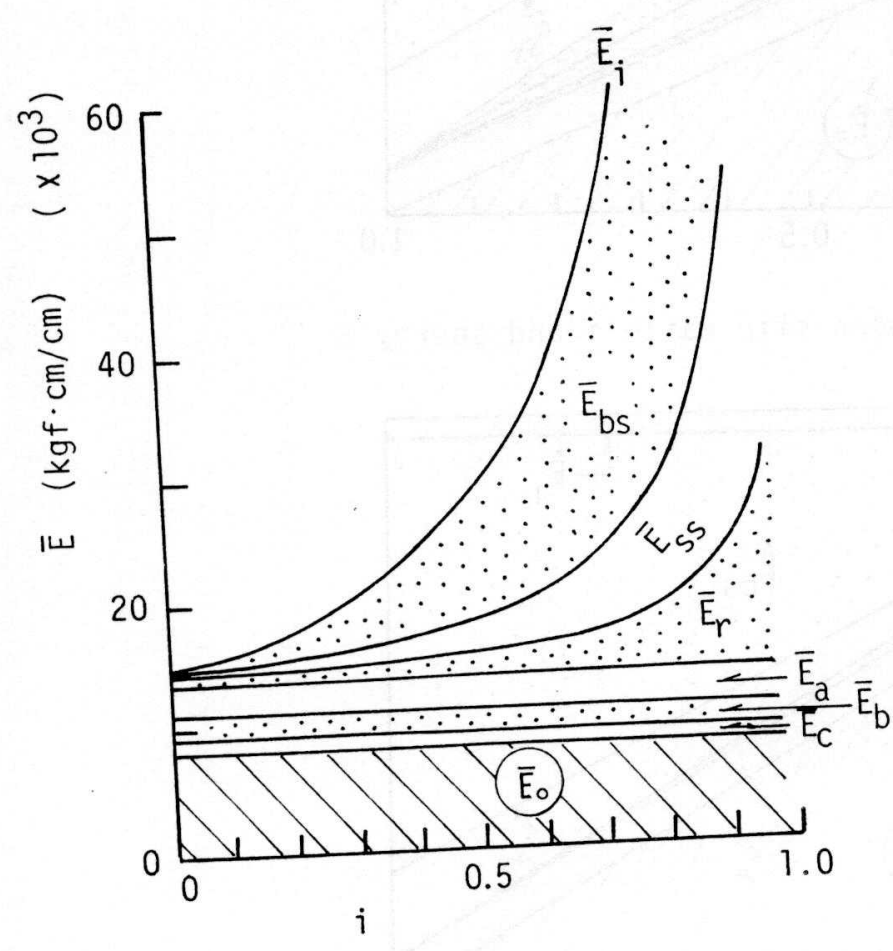

Fig. 2 Relationships between slip ratio $i$ and energy $\bar{E}$ (cohesive soil)

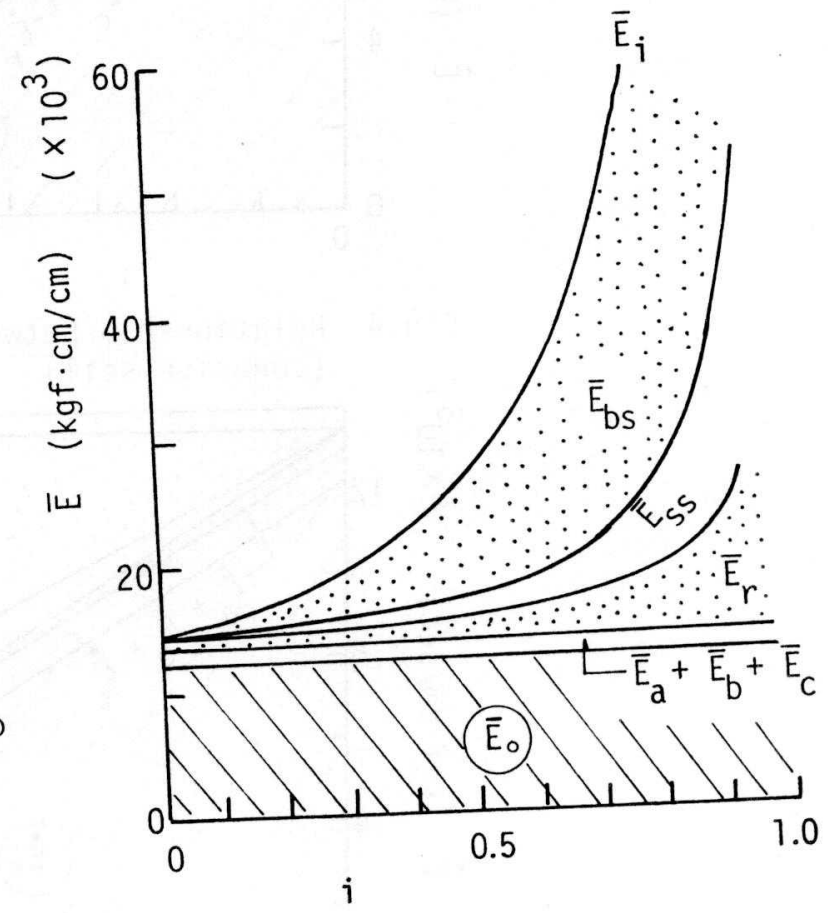

Fig. 3 Relationships between slip ratio $i$ and energy $\bar{E}$ (frictional soil) 
Figs. 2 and 3 show the relationship between slip ratio $i$ and energy $\bar{E}$ per unit travelling distance. It is evident that $\bar{E}_{j}$, $\bar{E}_{b s}$, E $\bar{E}_{s s}$ and $\bar{E}_{r}$ rapidly increase according to the increasing slip ratio. When $i=1.0$, the tractor is perfectly slipping and the most of input energy is spend for the bottom, side slip energies and the rotation energy.

Figs. 4 and 5 show the relationships between slip ratio $i$ and energy $\dot{E}$ per unit time. The input energy per unit time is constant regardless of slip ratio. When the slip ratio is small, the most of input energy changes to the bottom slip energy, side slip energy and others. The drawbar pull energy is 1 inearly decreased according the increasing the slip ratio.

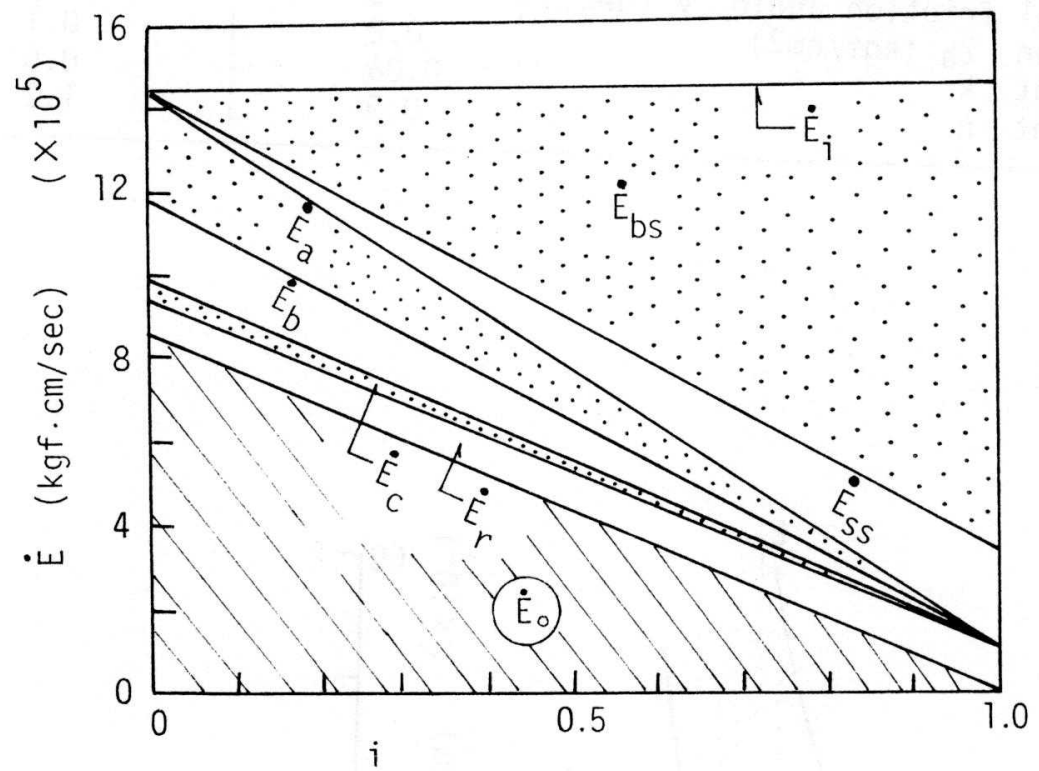

Fig.4 Relationship between slip ratio $i$ and eneray $\dot{E}$ (cohesive soil)

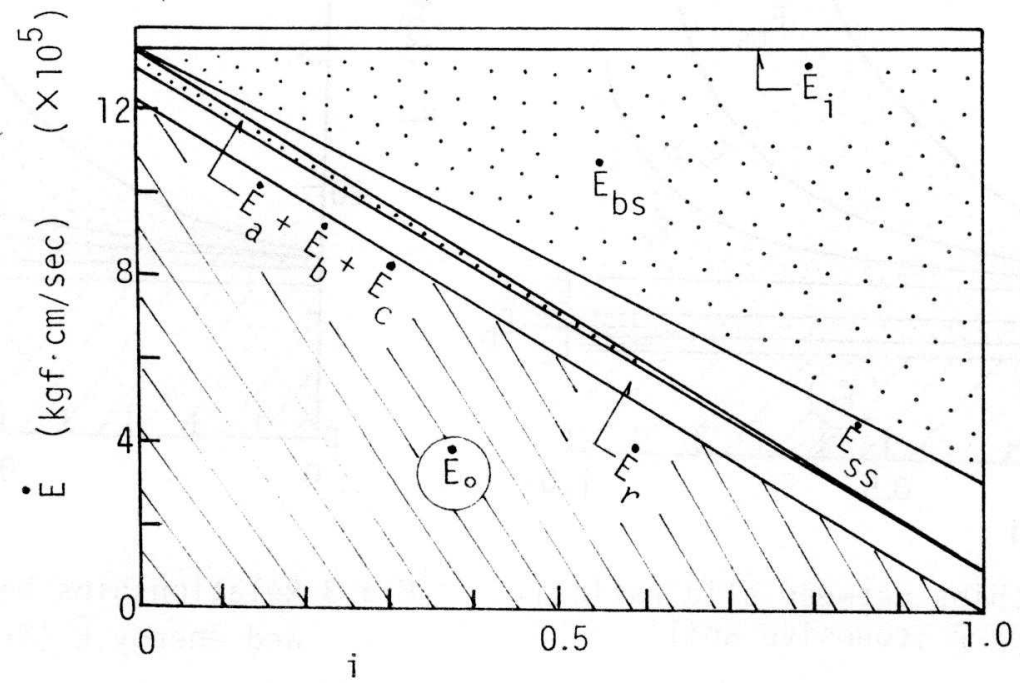

Fig. 5 Relationship between slip ratio $i$ and energy $\dot{E}$ (frictional soil) 


\section{CONCLUSIONS}

From the analysis of energy conservation between running gear and soil and the discussions on the results calculated, we can conclude as follows.

The six types of motion resistance acting on running gear of crawler tractor evaluated in terms of the output energy that can be developed in view of the track contact surface characteristics and soil properties. The energy conservation between the running gear and soil can be represented in terms of the energy per unit travelling distance and per unit time. The input energy per unit travelling distance, the bottom slip energy, the side slip energy and the rotation energy rapidly increase according to the increasing slip ratio. When the crawler is perfectly slipping, the most of the input energy is spend for the bottom slip energy, the side slip energy and the rotation energy. The input energy per unit time is constant regardless of the slip ratio. The most of the input energy changes to the drawbar pull energy for small slip ratio. The drawbar pull energy is linearly decreased according to the increasing the slip ratio. When the slip ratio is large, the most of input energy per unit time is lost in the bottom slip energy, the side slip energy and others.

\section{REFERENCES}

[1] Yong,R.N. and Webb,G.L.(1969) : Energie dissipation and drawbar pull prediction in soil-vehicle interaction, Proc. of 3rd Int. Conf., ISTVS, Vol.1, pp.93-142.

[2] Yong,R.N. and Fattah,E.A. (1975): Influence of contact characteristics on energy transfer and wheel performance on soft soil, Proc. of 7 th Int. Conf. ISTVS, Vol.2, pp.291-310.

[3] Muro,T., Omoto,K. and Nagira,A. (1989) : Traffic performance of a bul1dozer running on a weak terrain(Energy analysis), Proc. of JSCE, No.403/6 - 10, pp. 103-110 (Japanese).

[4] Kogure,K, Ohira,Y. and Yamaguchi,H.(1983): Prediction of sinkage and motion resistance of a tracked vehicle using plate penetration test, Jour. of Terramechanics, Vol.20, No.3/4, pp.121-128.

[5] Kogure,K., Ohira,Y. and Yamaguchi,H.(1982): A simplified method for the estimation of soil thrust exerted by a tracked vehicle, Jour. of Terramechanics, Vol.19, No.3, pp.165-181.

[6] Bekker,M.G.(1960) : Off-The-Road Locomotion, Research and Development in Terramechanics, The Univ. of Michigan Press, pp.32-35. 